\title{
Mortalidade infantil por causas evitáveis na Bahia, 2000-2012
}

\section{Child mortality by avoidable causes in Bahia, 2000-2012}

\section{Mortalidad infantil por causas evitables en Bahia, 2000-2012}

\author{
Lívia Teixeira Tavares | liviateixeiratavares@gmail.com \\ Faculdade Inspirar. Curitiba, PR, Brasil. \\ Tatiane Falcão dos Santos Albergaria | tatianefalcao@hotmail.com \\ Universidade Federal da Bahia. Salvador, BA, Brasil. \\ Marina de Aguiar Pires Guimarães |marinapguimaraes@gmail.com \\ Universidade Federal de Minas Gerais. Belo Horizonte, MG, Brasil. \\ Rhaine Borges Santos Pedreira | rhaineborges@gmail.com \\ Universidade Estadual do Sudoeste da Bahia. Jequié, BA, Brasil. \\ Elzo Pereira Pinto Junior | elzojr@hotmail.com \\ Universidade Federal da Bahia, Instituto de Saúde Coletiva. Salvador, BA, Brasil.
}

\section{Resumo}

Este artigo tem como objetivo analisar as causas e taxas de mortes evitáveis em crianças com menos de um ano de idade na Bahia no período de 2000 a 2012. Para isso, realizou-se um estudo ecológico de série temporal. Foram considerados os óbitos de crianças da faixa em questão, cujas mães residiam na Bahia naquele período. A classificação de evitabilidade dos óbitos seguiu a Lista brasileira de causas de mortes evitáveis por intervenções do Sistema Único de Saúde. Os dados foram extraídos dos sistemas de informação em saúde (Sistema de Informação sobre Mortalidade, Sistema de Informação sobre Nascidos Vivos). Apesar das reduções não só da taxa de mortalidade infantil geral como daquela por causas evitáveis no período estudado, ainda persistem altas proporções dessa última em menores de um ano de idade na Bahia. Destacaram-se a diminuição das taxas de mortalidade reduzíveis por adequada assistência ao recémnascido e o aumento das taxas de mortalidade reduzíveis por adequada atenção à mulher na gestação. Concluiu-se que a abordagem da evitabilidade é um potente avaliador da resolutividade dos serviços de saúde prestados à saúde materno-infantil.

Palavras-chaves: mortalidade infantil; sistema de informação; causas de morte; morte evitável; Sistema Único de Saúde. 


\begin{abstract}
This article aims to analyze the causes of avoidable death in children under one and their rates from 2000 to 2012 in Bahia. It is based on an ecological time series study which takes account the deaths of children under one whose mothers resided in Bahia during that period. The avoidable deaths classification followed the Lista brasileira de causas de mortes evitáveis por intervenções do Sistema Único de Saúde (Brazilian list of avoidable deaths causes due to Unified Health System interventions). The data were extracted from health information systems (Mortality Information System, Live Births Information System). Despite reductions in both overall infant mortality rate and death rate by avoidable causes during the study period, there are still high rates of avoidable deaths in children under one in Bahia. The decrease in avoidable mortality rates by appropriated assistance to the newborn and the increase in avoidable mortality rates by appropriated women care during pregnancy draw attention. We can conclude that the approach of the avoidable death causes is a potent evaluator of how to improve the health services supplied to maternal and child health.
\end{abstract}

Keywords: infant mortality; information system; causes of death; avoidable death; Unified Health System.

\title{
Resumen
}

Este artículo tiene el objetivo de analizar las causas y tasas de muertes evitables de niños menores de un año de edad en Bahia en el periodo de 2000 a 2012. Para eso fue realizado un estudio ecológico de serie temporal. Fueran consideradas las muertes de niños con edad inferior a un año, de los cuales las madres residían en Bahia en aquel periodo. La clasificación de la posibilidad de evitar las muertes siguió la Lista brasileira de causas de mortes evitáveis por intervenções do Sistema Único de Saúde (Lista brasileña de causas de muertes evitables por intervenciones del Sistema Único de Salud). Los datos fueron extraídos de los sistemas de información en salud (Sistema de Información al respecto de Mortalidad, Sistema de Información al respecto de Nacidos Vivos). A pesar de las reducciones en las tasas de mortalidad infantil general y por causas evitables en el periodo estudiado, aun persisten elevadas proporciones de muertes evitables en menores de un año de edad en Bahia. Sobresale la reducción de las tasas de mortalidad reducibles por apropriada asistencia al recién nacido y también el crecimiento de las tasas de mortalidad reducibles por apropriada atención a la mujer en la gestación. Concluimos que el enfoque de la evitabilidad es potente para evaluación de como mejorar la eficiencia de los servicios prestados a la salud maternoinfantil.

Palabras clave: mortalidad infantil; sistema de información; causas de muerte; muerte evitable; Sistema Único de Salud.

Contribuição dos autores:

Os autores do artigo tiveram igual participação na concepção e delineamento do estudo, na produção dos dados, na análise estatística, na preparação e redação do manuscrito e na revisão crítica final após a conclusão do trabalho.

Declaração de conflito de interesses: Os autores declaram não haver conflito de interesse.

Fontes de financiamentos: Não houve.

Considerações éticas: Por se tratar de estudos com dados secundários de domínio público e sem possibilidade de identificação dos sujeitos, dispensou-se a submissão do projeto à comitê de ética em pesquisa.

Agradecimento/Contribuições adicionais: Não houve.

Histórico do artigo: Submetido: 10.out.2015 | Aceito: 14.jun.2016 | Publicado: 30.set.2016.

Apresentação anterior: Não houve.

Licença CC BY-NC atribuição não comercial. Com essa licença é permitido acessar, baixar (download), copiar, imprimir, compartilhar, reutilizar e distribuir os artigos, desde que para uso não comercial e com a citação da fonte, conferindo os devidos créditos de autoria e menção à Reciis. Nesses casos, nenhuma permissão é necessária por parte dos autores ou dos editores. 


\section{Introdução}

A taxa de mortalidade infantil (TMI) é um indicador de saúde capaz de revelar as condições socioeconômicas de determinado território e a qualidade da assistência à saúde prestada à população materno-infantil ${ }^{1 .}$

Estimativas de organismos internacionais mostram uma redução significativa nas taxas de mortalidade na infância (menores de cinco anos) e nas taxas de mortalidade infantil (menores de um ano) nas últimas décadas. A TMI global reduziu de 63/1.00o Nascidos Vivos (NV), em 1990, para 34/1000 NV, em 2013. Na América Latina e Caribe, a queda no mesmo período foi de 43/1.00o NV para 15/1.0oo NV, comportamento semelhante ao visualizado no Brasil, cuja redução foi de 51/1.00o NV para 12/1.00oNV².

No cenário mundial, observam-se iniciativas que apoiam a redução da mortalidade infantil, especialmente em países pobres e em desenvolvimento. Entre elas, há um compromisso global, fomentado pela Organização das Nações Unidas (ONU), que aponta como um dos Objetivos de Desenvolvimento do Milênio (ODM) a redução da mortalidade infantil, ou seja, de crianças com menos de cinco anos de idade.

Apesar desse tema figurar na agenda da ONU, milhões de crianças ainda morrem por causas evitáveis no mundo. O conceito de morte evitável diz respeito à ocorrência desse evento quando poderia ser totalmente evitado pela adequada atenção à saúde, qualificando-o como indicador sensível à qualidade da assistência em saúde³. Doenças como pneumonia, diarreia e malária são consideradas causas de morte evitáveis e foram responsáveis, em 2013, por 51,8\% das mortes em crianças nos primeiros cinco anos de vida em todo o mundo ${ }^{2,4,5}$. No Brasil, apesar do declínio das taxas de mortes evitáveis em menores de um ano, o perfil de mortalidade infantil ainda é marcado por elevadas taxas desses óbitos ${ }^{6-8}$.

A necessidade de aprofundamento na temática de evitabilidade do óbito levou autores em diversos países a desenvolverem listas para classificação de causas de mortes evitáveis como ferramentas na prevenção e detecção de falhas na assistência à saúde ${ }^{3,9,10}$. No contexto brasileiro, pesquisadores, sob supervisão do Ministério da Saúde, construíram uma lista de causas de mortes evitáveis em menores de cinco anos por intervenções do Sistema Único de Saúde (SUS) ${ }^{11}$.

Pesquisas apontam desigualdades regionais na distribuição da assistência à saúde materno-infantil no Brasil. Dessa forma, o uso do critério de evitabilidade é um instrumento pertinente para auxiliar no monitoramento da qualidade, do acesso e da distribuição dos serviços de saúde em todas as regiões do país ${ }^{8,12}$.

Apesar da lista brasileira de causas de óbitos evitáveis em menores de cinco anos já ter sido publicada há alguns anos, ainda são escassas as pesquisas que levam em consideração esse conceito. Resultados de pesquisas nesse campo podem servir de subsídios para qualificação da assistência materno-infantil na Bahia. Nesse sentido, o presente estudo teve como objetivo analisar as causas e taxas de mortes evitáveis em menores de um ano na Bahia entre os anos 2000 e 2012.

\section{Método}

Trata-se de estudo ecológico de série temporal, com uso de dados secundários provenientes de sistemas de informação em saúde. Foram considerados os óbitos em crianças menores de um ano de idade, ocorridos na Bahia, entre janeiro de 2000 e dezembro de 2012, cujas mães residiam nesse mesmo estado. Optou-se por 2012 como o último ano da série, pois no momento da coleta dos dados esse ano representava o período mais recente com dados consolidados.

Para a extração dos dados, foram consultadas as Declarações de Óbitos do Sistema de Informação sobe Mortalidade (SIM). Também procedeu-se a consulta ao Sistema de Informação sobre Nascidos Vivos (SINASC) para a determinação do número de crianças nascidas na Bahia no período do estudo.

A classificação dos óbitos em causas evitáveis seguiu os códigos da Classificação Internacional de Doenças - CID 10 definidos pela versão atualizada da Lista de causas de mortes evitáveis por intervenções do Sistema 
Único de Saúde do Brasil, para crianças menores de cinco ano ${ }^{11}$. Essa lista divide as causas evitáveis em quatro grandes grupos: mortes reduzíveis por ações de imunoprevenção; mortes reduzíveis por adequada atenção à mulher na gestação, parto, feto e ao recém-nascido; mortes reduzíveis por ações adequadas de diagnóstico e tratamento; e mortes reduzíveis por ações adequadas de promoção à saúde, vinculadas a ações adequadas de atenção à saúde. O grupo de códigos de causas de mortes reduzíveis por adequada atenção à mulher na gestação, parto, feto e ao recém-nascido vem dividido em três subgrupos de causas: reduzíveis por adequada atenção à mulher na gestação; reduzíveis por adequada atenção à mulher no parto; e reduzíveis por adequada atenção ao feto e ao recém-nascido.

Para a análise do óbito em menores de um ano de idade foram construídos os seguintes indicadores: proporção de óbitos infantis por grupos de causas (causas evitáveis segundo grupos; mal definidas e demais causas); taxa de mortalidade infantil geral e segundo grupos de causas (causas evitáveis; mal definidas; não claramente evitáveis); proporção de óbitos por causas evitáveis (reduzíveis pelas ações de imunização; reduzíveis pela adequada atenção à mulher na gestação; reduzíveis por adequada atenção à mulher no parto; reduzíveis por adequada atenção ao recém-nascido; reduzíveis por ações adequadas de diagnóstico e tratamento; reduzíveis por ações de promoção da saúde) e taxa de mortalidade por causas evitáveis (reduzíveis pelas ações de imunização; reduzíveis pela adequada atenção à mulher na gestação; reduzíveis por adequada atenção à mulher no parto; reduzíveis por adequada atenção ao recém-nascido; reduzíveis por ações adequadas de diagnóstico e tratamento; reduzíveis por ações de promoção da saúde). Mais detalhes são apresentados no Quadro 1.

Quadro 1 - Indicadores de mortalidade infantil

\begin{tabular}{|c|c|c|c|}
\hline Variável & Descrição & Fórmula de cálculo & Fonte \\
\hline $\begin{array}{l}\text { Proporção de óbitos } \\
\text { infantis }\end{array}$ & $\begin{array}{l}\text { Percentual de óbitos em menores de } \\
\text { um ano de idade, na Bahia, segundo } \\
\text { grupos de causa (evitáveis, mal } \\
\text { definidas e não evitáveis) }\end{array}$ & $\begin{array}{l}\text { (Número de óbitos por grupo de } \\
\text { causa / número total de óbitos } \\
\text { infantis) x100 }\end{array}$ & SIM/SIM \\
\hline $\begin{array}{l}\text { Taxa de } \\
\text { mortalidade infantil }\end{array}$ & $\begin{array}{l}\text { Taxa de mortalidade em menores de } \\
\text { um ano, na Bahia, segundo grupos de } \\
\text { causa (evitáveis, mal definidas e não } \\
\text { evitáveis) e mortalidade geral }\end{array}$ & $\begin{array}{l}\text { Número de óbitos por grupo de } \\
\text { causa e óbitos totais / número de } \\
\text { nascidos vivos }\end{array}$ & SIM/SINASC \\
\hline $\begin{array}{l}\text { Proporção de óbitos } \\
\text { infantis por causas } \\
\text { evitáveis }\end{array}$ & $\begin{array}{l}\text { Percentual de óbitos evitáveis em } \\
\text { menores de um ano, na Bahia, } \\
\text { segundo grupos de causas (ações } \\
\text { de imunização, atenção à mulher } \\
\text { na gestação, no parto, ao recém- } \\
\text { nascido, ações de diagnóstico e } \\
\text { tratamento, promoção da saúde) }\end{array}$ & $\begin{array}{l}\text { (Número de óbitos por grupo de } \\
\text { causa evitável / número total de } \\
\text { óbitos por causas evitáveis) x100 }\end{array}$ & SIM/SIM \\
\hline $\begin{array}{l}\text { Taxa de } \\
\text { mortalidade por } \\
\text { causas evitáveis } \\
\text { em menores de um } \\
\text { ano de idade }\end{array}$ & $\begin{array}{l}\text { Taxa de mortalidade por causas } \\
\text { evitáveis em menores de um ano, } \\
\text { na Bahia, segundo grupos de causa } \\
\text { (ações de imunização, atenção à } \\
\text { mulher na gestação, no parto, ao } \\
\text { recém-nascido, ações de diagnóstico } \\
\text { e tratamento, promoção da saúde) }\end{array}$ & $\begin{array}{l}\text { Número de óbitos por grupo } \\
\text { de causa evitáveis / número de } \\
\text { nascidos vivos }\end{array}$ & SIM/SINASC \\
\hline $\begin{array}{l}\text { Variação percentual } \\
\text { de taxas e } \\
\text { proporção }\end{array}$ & $\begin{array}{l}\text { Variação das proporções e taxas de } \\
\text { mortalidade em menores de um ano } \\
\text { de idade }\end{array}$ & $\begin{array}{l}\left(\frac{V F-V I}{V I}\right) \times 100 \\
V F=\text { Valor final } \\
V I=\text { Valor inicial }\end{array}$ & - \\
\hline
\end{tabular}

Fonte: Elaborado pelos autores, 2015. 
A rotina de extração dos dados contou com a consulta às informações no site do Departamento de Informática do SUS - DATASUS e com o uso do TabNet, tabulador de dados que permite que eles sejam extraídos dados diretamente de web. Após a seleção das informações de interesse, foram geradas tabelas que, posteriormente, foram exportadas para planilhas do Microsoft Excel 2010. Já nas planilhas, foram calculados os indicadores e montados os gráficos e figuras com a síntese dos resultados. Ressalta-se ainda que este estudo não necessitou de submissão a Comitês de Ética em Pesquisa com seres humanos, uma vez que usou dados secundários de sistemas de informação em saúde disponibilizados na internet sem que houvesse a possibilidade de identificação pessoal dos sujeitos.

\section{Resultados}

A proporção de óbitos por causas evitáveis em menores de um ano de idade manteve-se com os maiores valores durante os anos da série temporal; entretanto, cabe destacar que as maiores variações ocorreram nas mortes por causas mal definidas, que caíram 82,2\%, e nas mortes por causas não evitáveis (demais causas), que aumentaram 100,42\%, como se vê na Figura 1.

A taxa de mortalidade infantil geral, no período estudado, variou de 26,63 para 17,01/ 1.00o NV (nascidos vivos), representando um decréscimo de 36,10\%. O coeficiente das mortes por causas mal definidas sofreu redução de 88,64\%, seguido por queda nas causas de mortes evitáveis (24,36\%). Diferentemente dos outros dois componentes, a taxa de mortalidade por causas não claramente evitáveis aumentou 28,06\% no período estudado, conforme é possível observar na Figura 2.

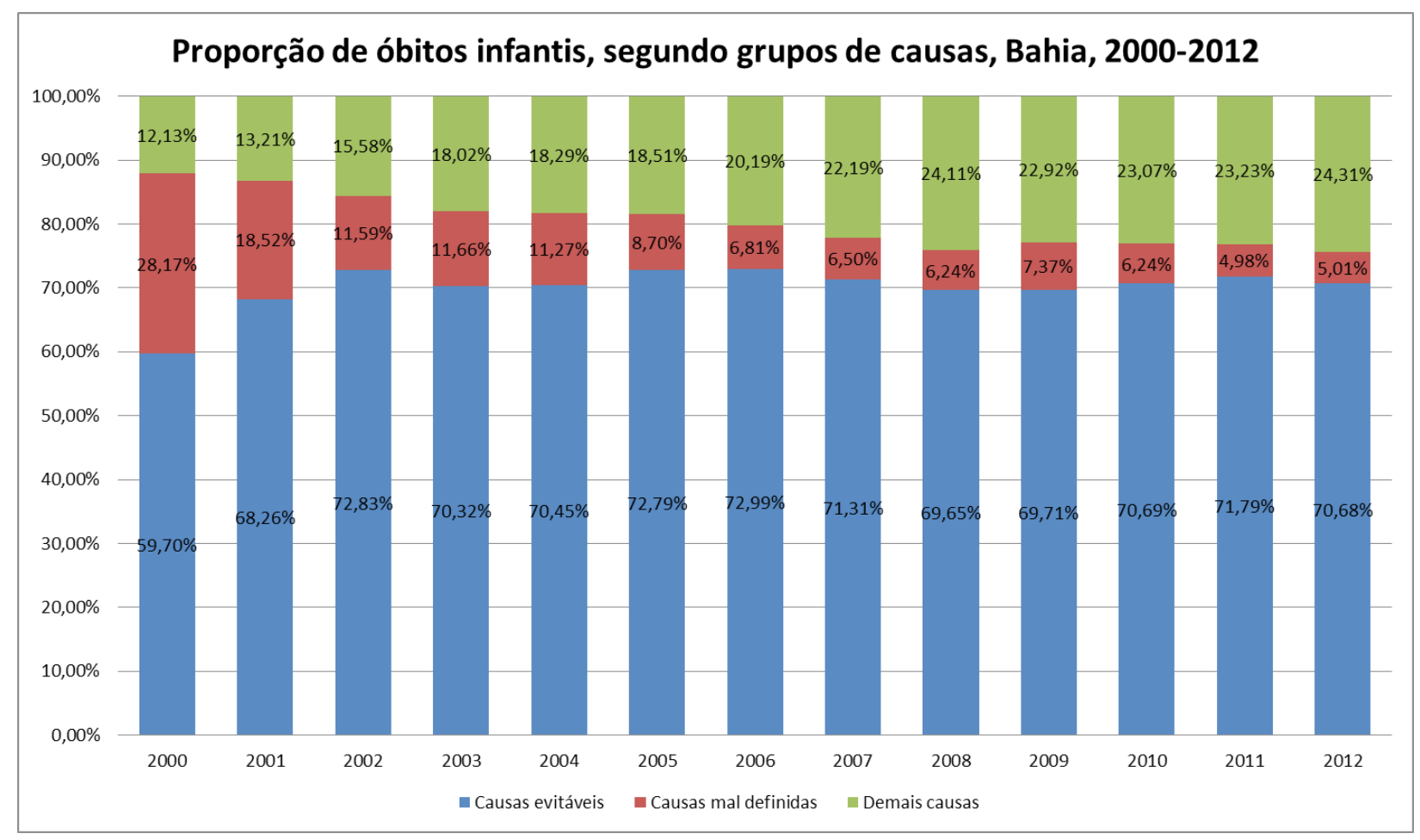

Figura 1 - Proporção de óbitos infantis, segundo grupo de causas, Bahia, 2000-2012 Fonte: SIM, DATASUS, 2015. 
Taxa de mortalidade infantil, segundo grupos de causas e mortalidade geral, Bahia, 2000-2012

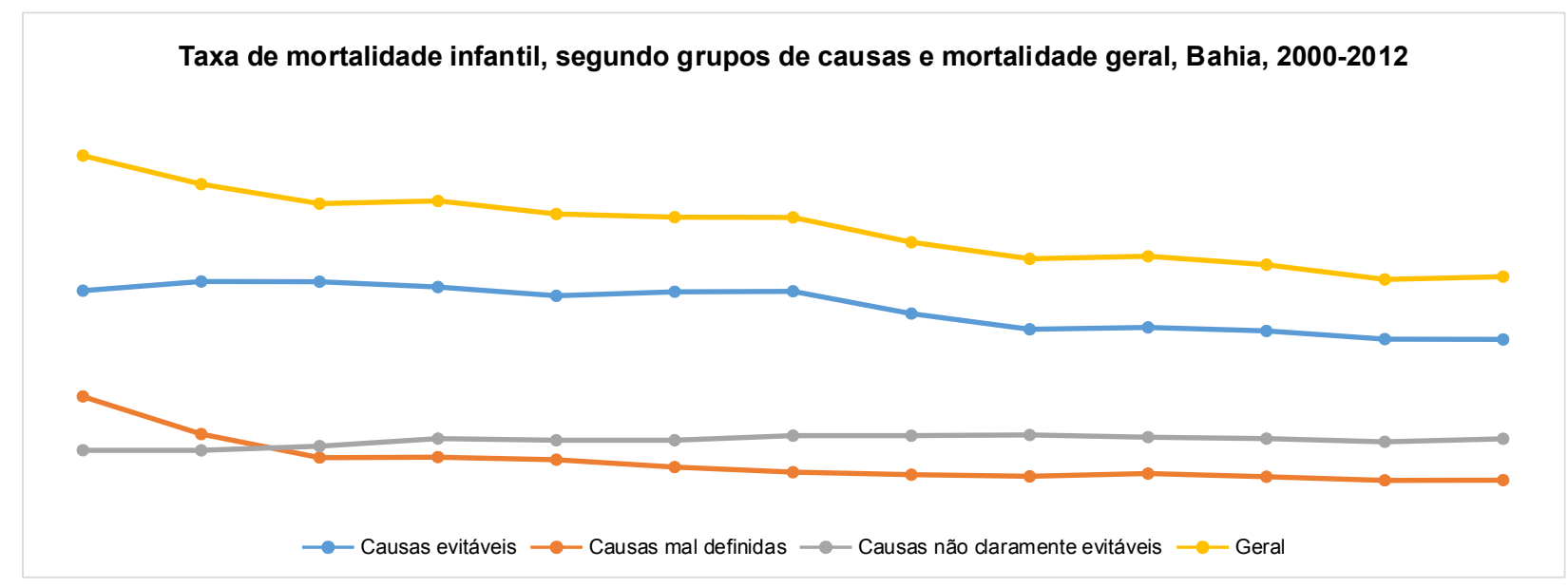

Figura 2 - Taxa de mortalidade infantil, segundo grupos de causas e mortalidade geral, Bahia, 2000-2012. Fonte: SIM, DATASUS, 2015.

Cabe salientar que as maiores proporções de óbito no período estudado são por causas reduzíveis por adequada assistência ao recém-nascido e por adequada atenção à mulher na gestação. Apesar de representarem os maiores percentuais ao final da série histórica, é importante ressaltar que a primeira foi reduzida enquanto a segunda cresceu. A proporção de mortes evitáveis por ações de imunização representou menos de $0,3 \%$, em todos os anos da série temporal e sua representação foi suprimida (Figura 3).

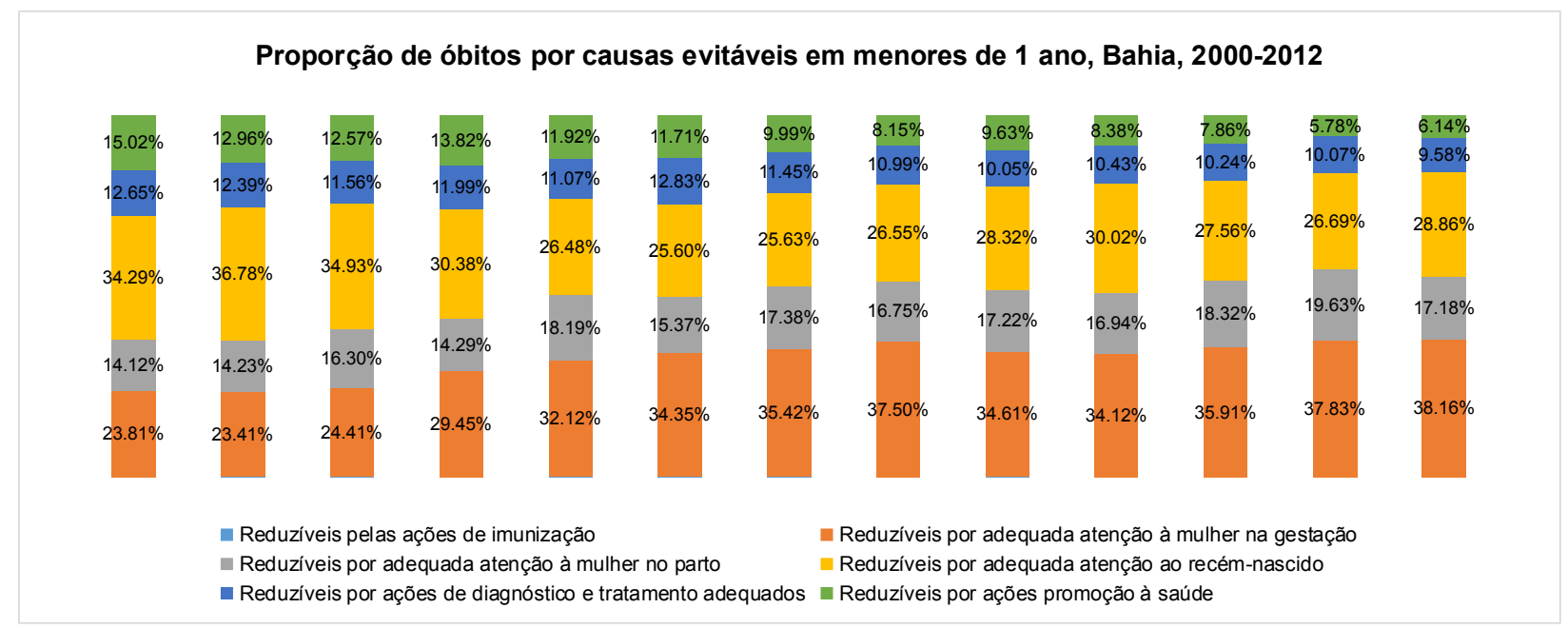

Figura 3 - Proporção de óbitos por causas evitáveis em menores de um ano de idade, Bahia, 2000-2012. Fonte: SIM, DATASUS, 2015.

Os coeficientes de mortalidade infantil por componente de causas evitáveis apresentaram comportamentos distintos. Houve uma redução na taxa de mortalidade infantil reduzível por ações de promoção à saúde, que diminuíram de 2,38 para 0,73/1.00o nascidos vivos, representando uma queda de 69,09\% em suas taxas. As taxas dos óbitos infantis reduzíveis por adequada atenção à mulher na gestação apresentaram aumento de 21,23\%, chegando a 4,59/1.00o nascidos vivos em 2012 (Figura 4). 
Taxa de mortalidade por causas evitáveis em menores de 1 ano, Bahia, 2000-2012

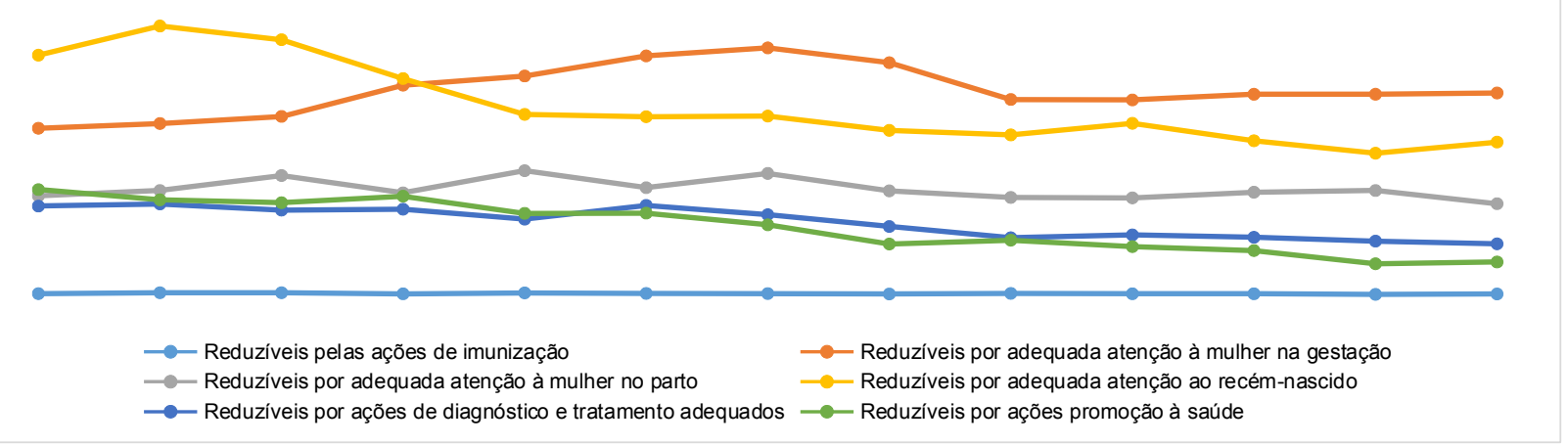

Figura 4 - Taxa de mortalidade por causas evitáveis em menores de um ano de idade, Bahia, 2000-2012 Fonte: SIM, DATASUS (2015).

\section{Discussão}

A análise da mortalidade infantil na Bahia, entre 2000 e 2012, revelou que a maior proporção de óbitos foi decorrente de causas evitáveis. Esse cenário encontrado na Bahia também foi visto em Maringá (PR), com 90,2\% de óbitos evitáveis em menores de cinco anos, e em Recife (PE), com 80\% de óbitos evitáveis em menores de um ano ${ }^{8,13}$. Estudo realizado com mortes ocorridas nos primeiros quatro anos de vida observou que parte dos óbitos evitáveis poderia ter sido inibida com recursos disponíveis atualmente, como a atenção pré-natal ${ }^{14}$. Os altos percentuais de mortes evitáveis sugerem problemas de acesso aos serviços de saúde, cobertura e qualidade da assistência prestada ${ }^{15}$. A avaliação das políticas e dos determinantes de saúde, na cidade de Úmbria (Itália), promoveu rastreio de fatores de risco e qualificação do seu sistema de saúde, exercendo impacto positivo sobre a redução da mortalidade evitável ${ }^{16}$.

A redução nos óbitos por causas mal definidas (CMD) na Bahia também foi visualizada num estudo de âmbito nacional que mostrou declínio desses eventos ${ }^{6}$. O Brasil apresentou, em 2010, um percentual de 8,6\% de óbitos por CMD em toda a população $0^{17}$. Complementando esse achado, notou-se aumento das proporções dos óbitos por causas não claramente evitáveis (demais causas) na Bahia e no Paraná, na cidade de Londrina, num estudo que comparou mortalidade infantil em dois biênios (2000/2001 e 2007/2008) e evidenciou aumento das mortes por causas não claramente evitáveis no último biênio, e redução do número de mortes por causas mal definidas. Isso pode indicar uma melhoria na qualidade da informação sobre causa da morte e sinalizar ampliação nos investimentos para o adequado diagnóstico e completude no preenchimento dessas declarações de óbitos pelos profissionais médicos ${ }^{7,17,18}$.

O estudo em que se baseia este artigo observou redução na taxa de mortalidade infantil (TMI) geral na Bahia no período analisado, corroborando o que a literatura aponta, ou seja, que tem ocorrido queda significativa na mortalidade infantil em todas as regiões brasileiras, com mudanças marcantes no Nordeste, região que historicamente apresenta elevados níveis da $\mathrm{TMI}^{19}$. A taxa de mortalidade em crianças menores de cinco anos de idade vem reduzindo no âmbito global e o Brasil está entre os 31 países que se encontram no caminho para atingir o objetivo 4, dos Objetivos de Desenvolvimento do Milênio (ODM) até $2015^{20}$.

Essas tendências indicam melhorias no acesso da população aos serviços primários em saúde através de programas como o Programa de Saúde da Família, e investimentos na saúde da criança, como a criação de comitês locais para a prevenção da mortalidade infantil e o programa Pacto pela Vida ${ }^{21}$. A maioria dos Comitês de Investigação do Óbito Infantil (CIOI) no Brasil encontra-se nas regiões Sul e Sudeste. Portanto, é importante destacar a necessidade de distribuir comitês esses em todo o território nacional, divulgar sua função e qualificar o trabalho desses grupos no enfrentamento da mortalidade infantil ${ }^{22}$. $\mathrm{O}$ aumento 
da despesa pública com a saúde, atenção em saúde voltada para o núcleo familiar, em especial para as crianças, acesso ao saneamento e abastecimento de água foram outras iniciativas que se associaram à diminuição da TMI no Brasil ${ }^{1,23,24}$.

Houve queda dos óbitos reduzíveis por adequada assistência ao recém-nascido e aumento das mortes por adequada atenção à mulher na gestação durante o período em análise concernente à Bahia. A redução dos óbitos por adequada assistência ao recém-nascido é resultado do aumento dos cuidados intensivos neonatais e com os recém-nascidos logo após o parto ${ }^{6}$. Já o aumento de óbitos evitáveis por adequada atenção à mulher na gestação e parto sugere obstáculos na assistência materno-infantil. Avaliação incompleta na admissão da gestante, dificuldade de acesso à maternidade e demora em iniciar a reanimação neonatal na sala de parto são falhas registradas na assistência à gestante e ao recém-nascido. A necessidade de qualificação do pré-natal e parto, formação profissional, valorização do planejamento reprodutivo e fortalecimento da atenção básica em saúde são primordiais nos investimentos em saúde $6,8,12,25$.

Os estudos com dados secundários dos sistemas de informação em saúde do SUS devem sempre considerar como limitação a qualidade no preenchimento de Declarações de Óbitos, que ainda está longe dos padrões desejados. Ainda assim, os sistemas de informação em saúde, especialmente o Sistema de Informação sobre Mortalidade têm sido aprimorados em todo o território brasileiro ${ }^{26}$. Apesar da limitação mencionada, a possibilidade do uso desses sistemas de informação permite que estudos com grande quantidade de sujeitos sejam realizados com baixo custo e num curto espaço de tempo, servindo de base para a gestão e o planejamento das ações voltadas para a melhoria das condições de saúde da população brasileira.

Os resultados do estudo que dá suporte a este artigo evidenciam que, no estado da Bahia, houve redução da mortalidade infantil por causas evitáveis, porém ainda são altos os índices de óbitos reduzíveis por adequada assistência ao recém-nascido e por adequada atenção à mulher na gestação.

Tendo em vista esse cenário, os esforços e investimentos para diminuir a TMI devem continuar através de uma melhor qualidade na assistência à saúde materno-infantil e fortalecimento dos comitês de investigação dos óbitos infantis. Aponta-se também a necessidade de continuar desenvolvendo ações preventivas, como o planejamento reprodutivo, e buscar o máximo de qualidade e resolutividade nos serviços de pré-natal, parto e puerpério.

\section{Referências}

1. Carvalho RAS, Santos VS, Melo CM. Desigualdades em saúde: condições de vida e mortalidade infantil em região do nordeste do Brasil. Rev Saúde Pública [Internet]. 2015 Jul [citado em 2016 Jul 26];49(5):1-9. Disponível em: http://www.scielo.br/scielo.php?script=sci_arttext\&pid=S003489102015000100202\&lng=en

2. United Nations Children's Fund. Committing to child survival: a promise renewed. Progress report 2014 [Internet]. New York, 2014. 104 p. [citado em 2016 Jul 26]. Disponível em: http://files.unicef.org/ publications/files/APR 2014 web_15Sept14.pdf

3. Malta DC, Duarte EC, Escalante JJC, Almeida MF, Sardinha LMV, Macário EM, et al. Causas de mortes evitáveis por ações efetivas dos serviços de saúde: uma revisão da literatura. Ciênc Saúde Coletiva [Internet]. 2007 [citado em 2016 Jul 26];12(3):765-76. doi: http://dx.doi.org/10.1590/S1413$\underline{81232007000300027}$

4. $\quad$ Organização das Nações Unidas. Relatório sobre os objetivos de desenvolvimento do milénio: 2013. Nova Iorque, 2013. 62 p. [citado em 2016 Jul 26]. Disponível em: https://www.unric.org/html/ portuguese/mdg/MDG-PT-2013.pdf

5. Liu L, Oza S, Hogan D, Perin J, Rudan I, Lawn JE, et al. Global, regional, and national causes of child mortality in 2000-13, with projections to inform post-2015 priorities: an updated systematic 
analysis. Lancet [Internet]. 2015 Jan [citado em 2016 Jul 26];385(9966):430-40. doi: 10.1016/S01406736(14)61698-6.

6. Malta DC, Sardinha LMV, Moura L, Lansky S, Leal MC, Szwarcwald CL, et al . Mortes evitáveis em menores de um ano, Brasil, 1997 a 2006: contribuições para a avaliação de desempenho do Sistema Único de Saúde. Cad Saúde Pública [Internet]. 2010 [citado em 2016 Jul 26];26(3):481-91. doi: http:// dx.doi.org/10.1590/S0102-311X2010000300006

7. Santos HG, Andrade SM, Silva AMR. Mortes infantis evitáveis por intervenções do Sistema Único de Saúde: comparação de duas coortes de nascimentos. Ciênc Saúde Coletiva [Internet]. 2014 [citado em 2016 Jul 26];19(3):907-16. doi: http://dx.doi.org/10.1590/1413-81232014193.01182013

8. Nascimento SG, Oliveira CM, Sposito V. Mortalidade infantil por causas evitáveis em uma cidade do Nordeste do Brasil. Rev Bras Enferm [Internet]. 2014 [citado em 2016 Jul 26];67(2):208-12. doi: http:// dx.doi.org/10.5935/0034-7167.20140027

9. Douglas MG, MaoY. Avoidable mortality in the United States and Canada, 1980-1996. Am J Public Health [Internet]. 2002 Sept [citado em 2016 Jul 26];92(9):1481-84. Disponível em: http://www.ncbi.nlm.nih. gov/pmc/articles/PMC1447265/

10. Simonato L, Ballard T, Bellini P. Avoidable mortality in Europe 1955-1994: a plea for prevention. J Epidemiol Community Health [Internet]. 1998 Oct [citado em 2016 Jul 26];52(10):624-30. Disponível em: http://jech.bmj.com/content/52/10/624.long

11. Malta DC, Duarte EC. Atualização da lista de causas de mortes evitáveis por intervenções do Sistema Único de Saúde do Brasil. Epidemiol Serv Saúde [Internet]. 2010 Apr-Jun [citado em 2016 Jul 26];19(2):173-76. Disponível em: http://scielo.iec.pa.gov.br/pdf/ess/v19n2/v19n2a10.pdf

12. Martins EF, Rezende EM, Lana FCF. Óbitos perinatais investigados e falhas na assistência hospitalar ao parto. Esc Anna Nery [Internet]. 2013 Jan-Mar [citado em 2016 Jul 26];17(1):38-45. doi: http://dx.doi. org/10.1590/S1414-81452013000100006

13. Jodas DA, Scochi MJ, Moura MB. Atendimento à criança e às mães: investigação do óbito evitável em menores de cinco anos. Rev Gaúcha Enferm [Internet]. 2011 Dec [citado em 2016 Jul 26]; 32(4):66975. doi: http://dx.doi.org/10.1590/S1983-14472011000400005

14. Santos IS, Matijasevich A, Barros AJD, Albernaz EP, Domingues MR, Valle NCJ, et al. Avoidable deaths in the first four years of life among children in the 2004 Pelotas (Brazil) birth cohort study. Cad Saúde Pública [Internet]. 2011 [citado em 2016 Jul 26]; 27(2):185-97. doi: http://dx.doi.org/10.1590/S0102$\underline{311 \times 2011001400007}$

15. Silva CMCD, Gomes KROG, Rocha OAMS. Validade, confiabilidade e evitabilidade da causa básica dos óbitos neonatais ocorridos em unidade de cuidados intensivos da Rede Norte-Nordeste de Saúde Perinatal. Cad Saúde Pública [Internet]. 2013 Mar [citado em 2016 Jul 26]; 29(3):547-56. doi: http:// dx.doi.org/10.1590/S0102-311X2013000300012

16. Stracci F, Bianconi F, Masanotti GM. Effect of healthcare on mortality: trends in avoidable mortality in Umbria, Italy, 1994-2009. Ann Ist Super Sanita [Internet]. 2013 [citado em 2016 Jul 26];49(2):113-23. doi: 10.4415/ANN_13_02_02.

17. França E, Teixeira R, IshitaniI L, Duncan BB, Cortez-Escalante JJ, Neto OLM, et al. Ill-Defined causes of death in Brazil: a redistribution method based on the investigation of such causes. Rev Saúde Pública [Internet]. 2014 Aug [citado em 2016 Jul 26];48(4):671-81. doi: http://dx.doi.org/10.1590/S0034$\underline{8910.2014048005146}$

18. Pacheco CP. Evolução da mortalidade infantil, segundo óbitos evitáveis: macrorregiões de saúde do estado de Santa Catarina, 1997-2008 [dissertação on line]. Faculdade de Saúde Pública: Universidade de São Paulo; 2010 [citado em 2016 Jul 26]. Disponível em: http://www.teses.usp.br/teses/ disponiveis/6/6132/tde-23022011-110058/pt-br.php

19. Barros FC, Matijasevich A, Requejo JH, Giugliani E, Maranhão AG, Monteiro CA, et al. Recent trends in maternal, newborn, and child health in Brazil: progress toward millennium development goals 4 and 5. Am J Public Health [Internet]. 2010 [citado em 2016 Jul 26];10:1877-89. doi: 10.2105/ AJPH.2010.196816.

20. Lozano R, Wang H, Foreman KJ, Rajaratnam JK, Naghavi M, Marcus JR, et al. Progress towards millennium development goals 4 and 5 on maternal and child mortality: an updated systematic analysis. Lancet [Internet]. 2011 [citado em 2016 Jul 26];378:1139-65. doi: 10.1016/S0140-6736(11)61337-8. 
21. Victora CG, Aquino EML, Leal MC. Maternal and child health in Brazil: progress and challenges. Lancet [Internet]. 2011 [citado em 2016 Jul 26];377:1863-76. doi: 10.1016/S0140-6736(11)60138-4

22. Venâncio SI, Paiva R.O processo de implantação dos comitês de investigação do óbito infantil no Estado de São Paulo. Rev Bras Saude Mater Infant [Internet]. 2010 [citado em 2016 Jul 26];10(3): 369-75. doi: http://dx.doi.org/10.1590/S1519-38292010000300010

23. Volpe FM, Abrantes MM, Capanema FD. The impact of changing health indicators on infant mortality rates in Brazil, 2000 and 2005. Rev Panam Salud Publica [Internet]. 2009 [citado em 2016 Jul 26];26(6):478-84. doi: http://dx.doi.org/10.1590/S1020-49892009001200002.

24. Lourenço EC, Guerra LM, Tuon RA, Silva SMCV, Ambrosano GMB, Corrente JE ,et al . Variáveis de impacto na queda da mortalidade infantil no Estado de São Paulo, Brasil, no período de 1998 a 2008. Ciênc Saúde Coletiva [Internet]. 2014 [citado em 2016 Jul 26];19(7):2055-62. doi: http://dx.doi. org/10.1590/1413-81232014197.18822013

25. Batista RV, Duarte EC , Sardinha LMV. Evolução da mortalidade infantil por causas evitáveis: série histórica 1997-2006, Distrito Federal. Comun Ciênc Saúde [Internet]. 2011 [citado em 2016 Jul 26];3:201-10. Disponível em: http://bvsms.saude.gov.br/bvs/artigos/evolucao mortalidade infantil.pdf

26. Rios MA, Anjos KF, Meira SS. Completude do sistema de informação sobre mortalidade por suicídio em idosos no estado da Bahia. J Bras Psiquiatr . [Internet]. 2013 [citado em 2016 Jul 26];62(2): 131-38. doi: http://dx.doi.org/10.1590/S0047-20852013000200006 